\title{
FATORES PROGNÓSTICOS ASSOCIADOS AO TRATAMENTO CIRÚRGICO DA MIELORRADICULOPATIA ESPONDILÓTICA CERVICAL
}

\author{
PROGNOSTIC FACTORS ASSOCIATED WITH SURGICAL TREATMENT OF CERVICAL \\ SPONDYLOTIC MYELORADICULOPATHY
}

\section{FACTORES PRONÓSTICOS ASOCIADOS CON EL TRATAMIENTO QUIRÚRGICO DE LA MIELORRADICULOPATÍA CERVICAL ESPONDILÓTICA}

Alexandre Meluzzl ${ }^{1}$ Mário Augusto Taricco², Roger Schimidth Brock1, Mário Rubem Pena Dias', Gilberto Nakaguawa1,

Vinícius Monteiro de Paula Guirado1, Manoel Jacobsen Teixeira ${ }^{3}$

\section{RESUMO}

Objetivo: Identificar os fatores clínicos dos indivíduos, fatores sociais, ambientais e dos exames de imagem que se correlacionam ao resultado final de melhora neurológica em pacientes submetidos ao tratamento cirúrgico da mielopatia espondilótica cervical. Métodos: A avaliação clínica foi quantificada pela escala deficitária da JOA. Analisamos 200 casos de mielorradiculopatia cervical, operados no HC-FMUSP, no período de janeiro de 1993 a janeiro de 2007. A média de segmento foi de 06 anos e 08 meses. A análise radiológica foi baseada nos critérios de instabilidade de White e scala de Kellgren. Resultados: Em 80\% houve melhora, 14\% estabilização e em 6\% piora do quadro neurológico. A piora neurológica não foi associada com nenhum fator clínico, ambiental ou de imagem. A melhora neurológica foi diretamente proporcional a menor idade na cirurgia, ausência de co-morbidade, sinal de Hoffman, atrofia muscular, hipersinal medular na RNM, menor período de evolução pré-operatório, melhor status neurológico pré-operatório e inversamente proporcional ao diâmetro AP do canal medular e multiplicidade de compressões. Identificou-se associação com o tabagismo. Mais de 70 anos, evolução superior a 24 meses, atrofia muscular, pontuação JOA igual ou inferior a sete pontos e diâmetro AP do canal inferior ou igual a seis mm não foram associado à melhora.

Descritores: Doenças da medula espinal ou mielopatia; Descompressão cirúrgica; Estenose espinal.

\begin{abstract}
Objective: Identify the individual, social, environmental clinical factors and also imaging studies which correlate to the final result of neurological improvement in patients undergoing surgical treatment of cervical spondylotic myelopathy. Methods: The clinical assessment was quantified by the deficit in JOA scale. We analyzed 200 cases of cervical myeloradiculopathy surgically treated in HC-FMUSP, from January 1993 to January 2007. The mean follow-up was 6 years and 8 months. The analysis was based on radiological criteria of instability by White and Kellgren scale. Results: $80 \%$ had improved, $14 \%$ stabilized and $6 \%$ had worsened. The neurological deterioration was not associated with any clinical, environmental or imaging factor. The neurological improvement was directly proportional to the lower age at surgery, absence of co-morbidity, Hoffman sign, muscular atrophy, spinal cord hyperintensity on MRI, the shortest period of preoperative evolution, better preoperative neurological status and was inversely proportional to the AP diameter of the spinal canal and to multiple cord compressions. An association with smoking was observed. Over 70 years of age, evolution superior to 24 months, muscle atrophy, JOA score equal to or less than seven points and AP canal diameter less than or equal to $6 \mathrm{~mm}$ were not associated with improvement.
\end{abstract}

Keywords: Spinal cord diseases; Decompression surgical; Spinal stenosis.

\section{RESUMEN}

Objetivo: Identificar los factores clínicos de los pacientes, factores sociales, ambientales y de exámenes de imagen que se correlacionan con el resultado final de mejoría neurológica en pacientes sometidos a tratamiento quirúrgico de la mielopatía cervical espondilótica. Métodos: La evaluación clínica fue cuantificada por la escala de JOA. Se analizaron 200 casos de mielorradiculopatía cervical, operados en el HC-FMUSP, desde enero de 1993 a enero de 2007. El promedio del segmento fue de 06 años y 08 meses. El análisis radiológico se basó en los criterios de inestabilidad de White y en la escala de Kellgren. Resultados: El 80\% había mejorado, el 14\% tuvo estabilización y el 6\% presentó deterioro del cuadro neurológico. El empeoramiento neurológico no se asoció con ningún factor clínico, ambiental ni de imagen. La mejoría neurológica fue directamente proporcional a edad menor para la cirugía, ausencia de comorbilidad, signo de Hoffman, atrofia muscular, hiperintensidad de la medula espinal en la RM, período más corto de la evolución preoperatoria, mejor estado neurológico preoperatorio, y siendo inversamente proporcional al diâmetro AP del canal espinal y a las compresiones múltiples. Identificada una asociación con el tabaquismo. Más de 70 años de edad, la evolución superior a 24 meses, la atrofia muscular, la puntuación JOA igual o inferior a siete puntos y el diámetro AP del canal menor o igual a seis $\mathrm{mm}$ no se asociaron con a mejoría.

Descriptores: Enfermedades de la médula espinal o mielopatía; Descompresión quirúrgica; Estenosis espinal.

1. Médico Neurocirurgião, Médico Pesquisador, Assistente do Grupo de Cirurgia da Coluna Vertebral, Hospital das Clínicas da Faculdade de Medicina da Universidade de São Paulo, SP, Brasil.

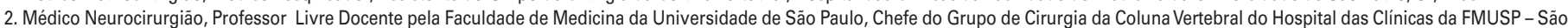
Paulo, SP, Brasil.

3. Médico Neurocirurgião, Professor Titular de Neurocirurgia do Hospital das Clínicas da FMUSP - São Paulo, SP, Brasil.

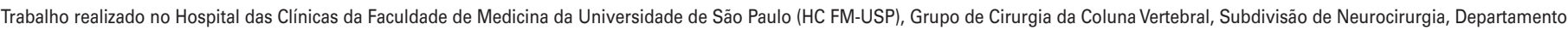
de Neurologia - São Paulo, SP, Brasil.

Correspondência: Av. Dr. Enéas de Carvalho Aguiar, 255 - 05403-000 - São Paulo, SP, Brasil. alemeluzzi@ ig.com.br 


\section{INTRODUÇÃO}

A espondilose cervical, termo que se refere às alterações degenerativas da coluna cervical, é uma condição degenerativa, idade relacionada, comum em indivíduos de populações industriais, em virtude do estilo de vida e a exposição a fatores ambientais e a trabalhos repetitivos onde se estima que entre 50\% e até $80 \%$ dos indivíduos apresentam no mínimo um episódio de dor cervical ao longo da vida, com ou sem componente radicular associado'. A doença degenerativa cervical é uma conseqüência natural do processo de envelhecimento, e em alguns indivíduos resulta em estenose do canal cervical, na maioria é assintomático, e em alguns tem expressão com consequência clínicas ${ }^{1}$.

A mielopatia espondilótica cervical, que é a condição mais grave da expressão clínica da espondilose, é a causa mais comum de disfunção medular adquirida em indivíduos com mais de 55 anos $^{2,3}$ e também a causa mais comum de tetraparesia e paraparesia espástica não traumática ${ }^{4,5}$. Aos 30 anos virtualmente todos os indivíduos podem apresentar alterações degenerativas em nível microscópico em sua coluna cervical, aos 40 muitos terão evidências de alterações degenerativas em radiografias ${ }^{6}$, condição que evolui progressivamente ao longo do tempo.

A mielopatia cervical se apresenta, com maior frequência, de maneira insidiosa, com curtos períodos de progressão sintomática seguidos por longos intervalos de relativa estabilidade de progressão dos sintomas ${ }^{5}$. Os sintomas, entretanto, em uma minoria dos casos, podem se desenvolver subitamente, especialmente com herniações discais agudas ou seguindo lesões por mecanismos de hiperextensão ou hiperflexão. Pode ainda se desenvolver em indivíduos com dor cervical axial ou quadros puros de radiculopatia, porém esta forma é incomum ${ }^{1,7}$.

Sinais e sintomas de mielopatia variam significativamente, de acordo com a localização e a gravidade da disfunção medular. Apesar de o canal medular ter diâmetro de 17-18mm entre C3 e C7, e poder acumular diversas estruturas ${ }^{8}$, alterações degenerativas tais como protrusão discal, ossificação do ligamento longitudinal posterior, osteófitos vertebrais, artrose facetaria, espessamento do ligamento amarelo e trauma, podem contribuir para estreitamento do canal medular e compressão da medula espinhal ${ }^{2,3}$. Se estas condições reduzem o diâmetro do canal a $13 \mathrm{~mm}$ ou menos ele será considerado estenótico ${ }^{9}$.

As alterações anatômicas locais secundárias a espondilose podem levar a síndromes clínicas bem definidas em virtude de compressões mecânicas e alterações inflamatórias das raízes nervosas, levando a quadros de radiculopatia ou a quadros de mielopatia secundários a compressões estáticas (estenose do canal) ou dinâmicas (instabilidade segmentar) da medula, ou ainda secundárias a alterações vasculares (isquemia ou ingurgitamento venoso) ou injúria celular (apoptose) da mesma, causando sintomas que podem variar desde dor até diferentes graus de comprometimento neurológico e incapacidade, de acordo com o grau de comprometimento das fibras axonais dos diferentes tratos medulares, ou do neurônio motor inferior $3,10-14$.

O estudo da história natural da espondilose cervical, e da síndrome clínica que pode decorrer desta, referida como mielorradiculopatia, aponta para um curso, muito provavelmente, de progressivo declínio das funções neurológicas ${ }^{4,14-17}$ embora possa haver estabilidade ou desaparecimento dos sintomas, como mostrado em alguns poucos estudos ${ }^{16-18}$

Há certo tipo de consenso que pacientes pouco ou moderadamente afetados podem ser cuidadosamente observados. Para pacientes com doença em evolução algum tipo de tratamento cirúrgico é indicado ${ }^{19}$.

O prognóstico para os pacientes com mielopatia é muito variável. Alguns estudos sugerem que o maior grau de incapacidade pré-operatório, maior número de níveis vertebrais envolvidos, sintomas de mielopatia com duração superior a 01 ano, déficit motor bilateral, área do canal inferior a $30 \mathrm{~mm}^{3}$, ausência do sinal de Lhermitte's e idade avançada tem um impacto negativo no prognóstico ${ }^{20}$.
Os fatores que podem ser associados com risco aumentado de alterações espondilóticas incluem trauma ocupacional repetido, carga axial freqüente, predisposição genética e síndrome de Down ${ }^{21-24}$. O tabagismo também tem sido associado com degeneração discal ${ }^{25}$, e também é um fator de risco para espondilose.

Os objetivos do tratamento são a eliminação dos sintomas, por métodos analgésicos e fisiátricos, com restauração e fortalecimento muscular, o que pode ser tipicamente alcançado com métodos conservativos, de acordo com protocolos de serviços diversos ${ }^{26}$. Em casos de dor radicular persistente e compressão medular sintomática, refratários ao tratamento conservador, a cirurgia está indicada ${ }^{27}$

Fortes evidências sugerem que a precoce realização da cirurgia (sintomas com início há menos de um ano) é associada à substancial melhora no prognóstico neurológico ${ }^{27-29 .}$

Assim a determinação dos fatores que afetam a evolução desta patologia e análise da evolução dos indivíduos submetidos a tratamento cirúrgico por diferentes técnicas em nosso meio é de extremo interesse científico e importante repercussão social.

\section{MATERIAL E MÉTODO}

Análise retrospectiva dos prontuários e exames de imagem (Radiografia simples neutra, em ântero-posterior e lateral, Radiografia dinâmica em lateral, em flexão e extensão, Ressonância magnética e eventualmente a Tomografia Computadorizada da coluna cervical), de 200 casos, com síndrome clínica de mielopatia ou mielorradiculopatia, que foram submetidos a tratamento cirúrgico da doença degenerativa da coluna cervical no Hospital das Clínicas da Faculdade de Medicina da Universidade de São Paulo, no período de março de 1993 a janeiro de 2007.

Utilizou-se a via anterior em 160 casos e a via posterior sem fusão em 40, em 133 casos a apresentação foi com quadro de mielopatia pura e em 67 casos, mielopatia associada à radiculopatia (mielorradiculopatia).

Na via anterior foi realizada a descompressão por discectomia, em um ou múltiplos níveis ou a corpectomia. Na via posterior utilizou-se a laminectomia sem fixação, a laminoplastia ou a arcocristectomia.

Excluiu-se os casos associados à artrite reumatóide, espondilite anquilosante, diabetes mellitus, alcoolismo, cirurgia prévia na coluna cervical, trauma cervical, segmento clínico inferior a 24 meses e dados incompletos no prontuário.

A avaliação clínica foi embasada no exame neurológico no momento do diagnóstico com padrão de mielopatia ou radiculopatia e quantificada pela escala da JOA (Japanese Ortopaedic Association) traduzida (Tabela 1). Os pacientes foram reavaliados, utilizando-se a mesma escala, do primeiro ao sétimo dias do pós-operatório (imediato) e na última avaliação ambulatorial, com um mínimo de 24 meses (tardia) quando utilizamos a taxa de recuperação (JOA) como referência (Tabela 2).

A avaliação radiográfica consistiu na observação do alinhamento, presença ou não de lordose fisiológica, retificação ou inversão (cifotização) e ântero-listese e retro-listese dos níveis adjacentes à fusão. A lordose cervical foi avaliada na radiografia em perfil neutro, traçando-se uma linha perpendicular ao platô inferior de C2 e platô superior de C7, medida ainda realizada nas incidências em perfil em flexão e extensão. Nas três situações a medida entre cortical póstero-inferior da vértebra superior à cortical póstero-superior da vértebra inferior dos níveis adjacentes a artrodese. Uma listese maior que $02 \mathrm{~mm}$ no neutro ou uma mudança maior que $02 \mathrm{~mm}$ nas distâncias dinâmicas definiram instabilidade ${ }^{30}$.

Todos os pacientes com instabilidade no exame radiológico, estático ou dinâmico, foram submetidos à fixação interna com placa e parafusos.

A Ressonância magnética foi solicitada no pré-operatório em todos os casos, e novamente solicitada quando da presença de novos sintomas clínicos ou nos casos sem melhora neurológica precoce após o tratamento cirúrgico. Tardiamente foi solicitada em todos os casos. Onde havia evidências de calcificações ligamentares ou articulares a Tomografia computadorizada complementou o estudo radiológico, para fins de diagnóstico acurado e planejamento cirúrgico. 
Tabela 1. Escala da JOA para Mielopatia Cervical - 0 a 17 Pontos.

\begin{tabular}{|c|c|c|}
\hline $\mathbf{I}$ & Função motora no membro superior & \\
\hline & Impossível comer com colher ou abotoar camisa & 0 \\
\hline & Possivel comer com colher, impossível abotoar camisa & 1 \\
\hline & Possível abotoar camisa, com grande dificuldade & 2 \\
\hline & Possível abotoar camisa, com dificuldade & 3 \\
\hline & Normal & 4 \\
\hline \multirow[t]{6}{*}{ II } & Função motora no membro inferior & \\
\hline & Impossível & 0 \\
\hline & Necessita bengala ou auxílio em solo plano & 1 \\
\hline & Necessita auxílio em escadas & 2 \\
\hline & Anda sem auxílio, mas lentamente & 3 \\
\hline & Normal & 4 \\
\hline \multirow[t]{13}{*}{ III } & Função sensitiva & \\
\hline & MEMBRO SUPERIOR & \\
\hline & Distúrbio sensitivo aparente & 0 \\
\hline & Distúrbio sensitivo mínimo & 1 \\
\hline & Normal & 2 \\
\hline & MEMBRO INFERIOR & \\
\hline & Distúrbio sensitivo aparente & 0 \\
\hline & Distúrbio sensitivo mínimo & 1 \\
\hline & Normal & 2 \\
\hline & TRONCO & \\
\hline & Distúrbio sensitivo aparente & 0 \\
\hline & Distúrbio sensitivo mínimo & 1 \\
\hline & Normal & 2 \\
\hline \multirow[t]{5}{*}{ IV } & Função vesical & \\
\hline & Retenção urinária ou incontinência & 0 \\
\hline & Sensação de retenção/perda de pequeno fluxo & 1 \\
\hline & Retenção urinária e/ou polaciúria & 2 \\
\hline & Normal & 3 \\
\hline
\end{tabular}

Tabela 2. Taxa de recuperação (JOA).

Taxa de recuperação $(\%)=($ JOA Pós-op - JOA Pré-op) X 100

$$
17 \text { - JOA Pré-op }
$$

Observou-se múltiplas variáveis, qualitativas e quantitativas, associadas aos indivíduos, aos procedimentos e períodos, como o início do quadro clínico (sinais e sintomas), exames de imagem, modalidades cirúrgicas, evolução clínica e pontuação segundo a escala da JOA nos períodos pré-operatório e pós, índice de recuperação, intervalo de tempo entre os eventos, fatores ambientais associados e outros.

\section{RESULTADOS}

Apresentamos a seguir o resultado da análise.

\section{Sexo}

140 pacientes do sexo masculino e 60 feminino.

\section{Atividade laboral}

Tabela 3. Atividade laboral e distribuição.

\begin{tabular}{c|c|c}
\hline Atividade laboral & $\mathbf{n}$ & $\%$ \\
\hline Braçal & 59 & 29,5 \\
\hline Outro & 68 & 34 \\
\hline Técnico & 21 & 10,5 \\
\hline Liberal & 16 & 8 \\
\hline Aposentado & 36 & 18 \\
\hline Braçal & Lavrador, Ajudante geral, Ajudante de construção civil \\
\hline \multirow{2}{*}{ Outro } & $\begin{array}{c}\text { Pintor, Pedreiro, Carpinteiro, Cabeleireira, Do lar, Doméstica, } \\
\text { Motorista, Zelador, Mecânico }\end{array}$ \\
\hline \multirow{2}{*}{ Técnico } & $\begin{array}{c}\text { Auxiliar de Enfermagem, Metalúrgico, Escriturário, Técn. } \\
\text { Mecânico, Comerciário }\end{array}$ \\
\hline Liberal & Professor, Advogado, Dentista, Comerciante. \\
\hline Aposentado & Não informado atividade prévia. \\
\hline
\end{tabular}

Observamos maior incidência da doença nos grupos que exercem atividades laborativas que exigem maior atividade física com amior carga axial e executam trabalhos repetitivos.

\section{Idade no momento da cirurgia}

A idade variou entre um mínimo de 30 anos e um máximo de 84 anos, com média aritmética de 52,41 $\pm 12,23$, mediana e modo de 50 anos (Tabela 4).

Tabela 4. Idade dos pacientes no momento da cirurgia e média da pontuação na escala da JOA.

\begin{tabular}{c|c|c|c|c}
\hline Idade & $\mathbf{f}$ & JOA pré & JOA Pós & JOA Tardio \\
\hline $30+40$ & 30 & 9,5 & 13,66 & 13,3 \\
\hline $40+50$ & 68 & 9,07 & 12,83 & 12,39 \\
\hline $50+60$ & 45 & 10,73 & 12,66 & 12,91 \\
\hline $60+70$ & 38 & 9,78 & 12,02 & 12,5 \\
\hline $70+75$ & 19 & 8,89 & 10,84 & 10,57 \\
\hline$\sum$ & 200 & $X$ & $X^{2}$ & $X^{2}$
\end{tabular}

nível de significância $\alpha=0,001$ e z crítico 3,29 $/ \delta=2,48 / X=9,63$

$\mathrm{X}-\mathrm{X}^{1}=4,03 / \mathrm{X}-\mathrm{X}^{2}=3,67$

$X-X^{1}=3,2 / X-X^{2}=2,76$

$X-X^{1}=3,03 / X-X^{2}=3,28$

$X-X^{1}=2,39 / X-X^{2}=2,87$

$X-X^{1}=1,21 / X-X^{2}=0,94$

$4,03 / 2,48 / 5,47=4,03 / 0,45=8,95 / / / / 3,67 / 2,48 / 5,47=3,67 / 0,45=8,15$

$3,2 / 2,48 / 8,24=3,2 / 0,3=10,66 / / / / 2,76 / 2,48 / 8,24=2,76 / 0,3=9,2$

$3,03 / 2,48 / 6,7=3,03 / 0,37=8,18 / / / / 3,28 / 2,48 / 6,7=3,28 / 0,37=8,86$

$2,39 / 2,48 / 6,16=2,39 / 0,4=5,97 / / / / 2,87 / 2,48 / 6,16=2,87 / 0,4=7,17$

$1,21 / 2,48 / 4,35=1,21 / 0,57=2,12 / / / / 0,94 / 2,48 / 4,35=0,94 / 0,57=1,64$.

Tabela 5. Idade dos pacientes no momento da cirurgia e Taxa de recuperação precoce e tardia da JOA.

\begin{tabular}{c|c|c|c}
\hline Idade & $\mathbf{f}$ & TX Rec Pré & TX Rec Tard \\
\hline $30+40$ & 30 & 55,40 & 50,15 \\
\hline $40+50$ & 68 & 47,60 & 40,87 \\
\hline $50+60$ & 45 & 31,31 & 35,76 \\
\hline $60+70$ & 38 & 30,98 & 39,74 \\
\hline $70+75$ & 19 & 24,81 & 17,20 \\
\hline$\Sigma$ & 200 & $X$ & $X^{2}$ \\
\hline
\end{tabular}

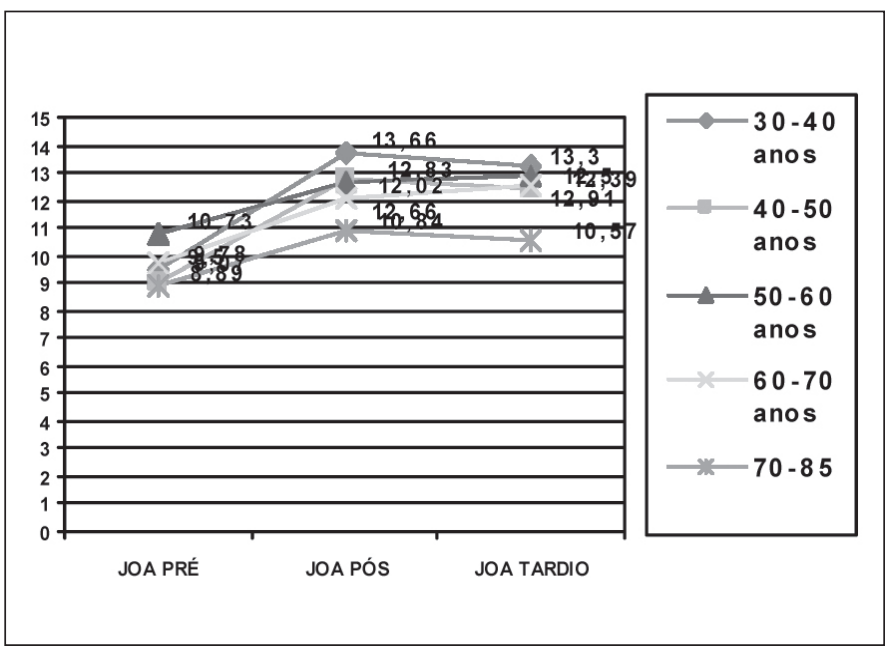

Figura 1. Evolução Operatória segundo a idade dos pacientes. 
A análise estatística das médias de pontuação obtidas pela aplicação da escala da JOA nos períodos pré-operatório, pós-operatório imediato e pós-operatório tardio, em função da idade no momento da apresentação clínica, evidencia incremento na pontuação pré-pós operatória, com significância estatística para $\alpha=$ 0,001, nos grupos etários compreendidos no intervalo de idade de vida entre 30 e 60 anos, sendo observada associação inversa entre idade e melhora na pontuação da escala da JOA.. No grupo com 19 pacientes e idades superiores á 70 anos, não houve incremento estatisticamente significativo, na pontuação, segundo a escala da JOA, entre os períodos pré e pós-operatório, sendo que, para este grupo, a cirurgia não produz o resultado esperado, sem associação positiva ou negativa no resultado final (Tabela 6).

Tabela 6. Grupos etários e suas correlações com pontuação JOA Pré Op. e período de evolução Pré Op.

\begin{tabular}{c|c|c|c}
\hline Idade & $\mathbf{f}$ & JOA Pré-0p & Período Evol. Pré-0p \\
\hline $30+40$ & 30 & 9,5 & 12,5 \\
\hline $40+50$ & 68 & 9,07 & 16 \\
\hline $50+60$ & 45 & 10,73 & 22,48 \\
\hline $60+70$ & 38 & 9,78 & 23 \\
\hline $70+$ & 19 & 8,89 & 19,52 \\
\hline
\end{tabular}

Intervalo de confiança

\begin{tabular}{c|l}
\hline $30-40$ & $9,63 \pm 1,49(8,14-11,12)$ e $18,6 \pm 9,14(9,46-27,74)$ \\
\hline $40-50$ & $9,63 \pm 0,99(8,64-10,62)$ e $18,6 \pm 6,06(12,54-24,66)$ \\
\hline $50-60$ & $9,63 \pm 1,21(8,42-10,84)$ e $18,6 \pm 7,46(11,14-26,06)$ \\
\hline $60-70$ & $9,63 \pm 1,32(8,31-10,95)$ e $18,6 \pm 8,11(10,49-26,71)$ \\
\hline $70-$ & $9,63 \pm 1,87(7,76-11,5)$ e $18,6 \pm 11,49(7,11-30,09)$ \\
\hline
\end{tabular}

Não foi constatado diferença significativa no período de evolução pré-operatório da doença, em cada grupo etário, que pudesse influenciar no resultado final.

\section{Fatores ambientais}

Em 96 casos $(46,5 \%)$ houve associação com tabagismo, em 14 casos (7\%) houve associação com doença ortopédica de alguma natureza, como assimetria no tamanho dos membros inferiores, escoliose da coluna vertebral, artrose em membros inferiores e outros. Outras co-morbidades encontradas foram: seqüela de doença neurológica (1), sequela de poliomielite (1), disrafismo espinhal (1) hipertensão arterial sistêmica (30), cardiopatia (11), hipotireoidismo (2), insuficiência vascular periférica (2), obesidade (3), dislipidemia (2) e outros (Tabela 7).

Tabela 7. Co-morbidades e evolução operatória.

\begin{tabular}{c|c|c|c|c}
\hline & $\mathbf{f}$ & JOA Pré & JOA Pós & JOA Tardio \\
\hline $\operatorname{Sim}$ & 41 & 9,45 & 11,42 & 11,37 \\
\hline Não & 159 & 9,6 & 12,87 & 12,81 \\
\hline
\end{tabular}

$X-X^{1}=1,79 / X-X^{2}=1,74$

$X-X^{1}=3,24 / X-X^{2}=3,18$

$1,79 / 2,48 / 6,4=1,79 / 0,387=4,62 / / / / 1,74 / 2,48 / 6,4=1,74 / 0,387=4,49$

$3,24 / 2,48 / 12,6=3,24 / 0,196=16,53 / / / / 3,18 / 2,48 / 12,6=3,18 / 0,196=16,22$

nível de significância $\alpha=0,001$ e z crítico 3,29 $/ \delta=2,48 / X=9,63$.

Tabela 8. Co-morbidades e idade e período de evolução pré-operatória.

\begin{tabular}{c|c|c|c}
\hline & $\mathbf{f}$ & Idade & Períod. Evol. Pré-Op \\
\hline $\operatorname{Sim}$ & 43 & 59,77 & 19,3 \\
\hline Não & 157 & 50,67 & 18,6 \\
\hline
\end{tabular}

\section{Intervalo de confiança}

\begin{tabular}{c|c}
\hline SIM & $(46,13-58,69)$ e $(10,79-26,41)$ \\
\hline NÃO & $(49,22-55,60)$ e $(14,63-22,56)$ \\
\hline
\end{tabular}

Distribuição dos pacientes em dois grupos, de acordo com a presença ou não de co-morbidades. A análise dos dados das médias de pontuação obtidas na aplicação da escala da JOA, nos períodos pré-operatório, pós-operatório imediato e pós-operatório tardio, evidencia incremento estatisticamente significativo, com $\alpha$ $=0,001$, entre os períodos pré-pós operatórios, nos dois grupos, rejeitando-se a hipótese de que os pacientes com co-morbidades não melhoram.

Há, entretanto evidente melhor desempenho do grupo sem co-morbidades, anotando melhores escores pós-operatórios, evidenciando associação negativa entre co-morbidade e desempenho cirúrgico, segundo a escala da JOA.

Há notadamente diferenças, com nível de significância estatístico $\alpha=0,001$, entre as médias de idade dos grupos, sendo no grupo onde há co-morbidades associadas, superiores, locando-se fora do valor superior do intervalo de confiança ( $n=43, \alpha=0,001$, $\delta=2,48$ e $X=9,63$ ), sendo que o fator idade tem correlação inversamente proporcional ao incremento pós-cirúrgico da pontuação obtida pela escala da JOA.

Assim o fator co-morbidade contribui para pior desempenho pós-cirúrgico, associado ao fator idade, ambos com relação inversa.

\section{Sinal de Hoffman}

O sinal de Hoffman, que indica lesão dos tratos piramidais longos, ao nível cervical, esteve presente em 184 pacientes (92\%) no exame clínico pré-operatório, gerando um grupo de pacientes com predominância acentuada sobre aqueles 16 que não apresentavam (8\%), uma vez que este é usado como parâmetro para a indicação do tratamento cirúrgico no serviço (Tabelas 9 e 10).

Tabela 9. Presença do sinal de Hoffman e evolução pós-operatória.

\begin{tabular}{c|c|c|c|c}
\hline & $\mathbf{f}$ & JOA Pré & JOA Pós & JOA Tardio \\
\hline $\operatorname{Sim}$ & 184 & 9,46 & 12,35 & 12,26 \\
\hline Não & 16 & 11,5 & 15,12 & 15,18 \\
\hline
\end{tabular}

$X-X^{1}=2,72 / X-X^{2}=2,63$

$X-X^{1}=5,49 / X-X^{2}=5,55$

$2,72 / 2,48 / 13,56=2,72 / 0,209=13,01 / / / / 2,63 / 2,48 / 2,89=2,63 / 0,209=12,58$ $5,49 / 2,48 / 4=5,49 / 0,62=8,85 / / / / 5,55 / 2,48 / 4=5,55 / 0,62=8,95$ nível de significância $\alpha=0,001$ e z crítico 3,29 $/ \delta=2,48 / X=9,63$

Tabela 10. Idade e período de evolução pré-operatório.

\begin{tabular}{c|c|c|c}
\hline & $\mathbf{f}$ & Idade & Períod. Evol. Pré Op. \\
\hline $\operatorname{Sim}$ & 184 & 52,42 & 18,81 \\
\hline Não & 16 & 52,25 & 16,18 \\
\hline
\end{tabular}

\section{Intervalo de confiança}

\begin{tabular}{c|l}
\hline SIM & $52,41 \pm 2,96(49,45-55,37)$ e $18,6 \pm 3,68(14,92-22,28)$ \\
\hline NÃO & $52,41 \pm 10,05(42,36-62,46)$ e $18,6 \pm 12,5(6,1-31,1)$ \\
\hline
\end{tabular}

Distribuição dos pacientes segundo a presença do sinal de Hoffman no exame clínico pré-operatório, agrupados em dois, segundo a presença ou não. A análise estatística das médias de pontuação obtidas pela aplicação da escala da JOA nos períodos pré-operatório, pós-operatório imediato e pósoperatório tardio evidencia incremento na pontuação pré-pós operatória, com significância estatística para $\alpha=0,001$, nos dois grupos, notadamente com melhor desempenho do grupo onde o sinal não está presente, observando-se associação inversa entre o sinal e melhora clínica, sem exclusão da melhora pós-operatória 
Atrofia muscular (Tabelas 11 e 12)

Esta indica lesão do neurônio motor inferior, na medula cervical. (Tabela 11)

Tabela 11. Presença de atrofia muscular e evolução operatória.

\begin{tabular}{c|c|c|c|c}
\hline & $\mathbf{f}$ & JOA Pré & JOA Pós & JOA Tardio \\
\hline $\operatorname{Sim}$ & 30 & 8,26 & 8,96 & 9,13 \\
\hline Não & 170 & 9,87 & 13,21 & 13,08 \\
\hline
\end{tabular}

$X-X^{1}=-0,67 / X-X^{2}=-0,5$

$X-X^{1}=3,58 / X-X^{2}=3,45$

$-0,67 / 2,48 / 5,47=-0,67 / 0,453=-1,47 / / / /-0,5 / 2,48 / 5,47=-0,5 / 0,453=-1,1$

$3,58 / 2,48 / 13,03=3,58 / 0,19=18,84 / / / / 3,45 / 2,48 / 13,03=3,45 / 0,19=18,15$

nível de significância $\alpha=0,001$ e z crítico 3,29 $/ \delta=2,48 / X=9,63$

Tabela 12. Idade e período de evolução pré-operatório.

\begin{tabular}{c|c|c|c}
\hline & $\mathbf{f}$ & Idade & Períod. Evol. Pré Po. \\
\hline $\operatorname{Sim}$ & 30 & 54,73 & 25,66 \\
\hline Não & 170 & 52 & 17,36 \\
\hline
\end{tabular}

Distribuição dos pacientes segundo a presença de atrofia muscular no exame clínico pré-operatório, agrupados segundo a observação ou não. A análise estatística das médias de pontuação obtidas pela aplicação da escala da JOA nos períodos pré-operatório, pós-operatório imediato e pós-operatório tardio evidencia incremento na pontuação pós-operatória, com significância estatística para $\alpha=0,001$, somente no grupo onde a atrofia muscular não se faz presente.

No grupo onde atrofia muscular é presente não há evidência estatística de incremento na pontuação pré-pós operatória, segundo a escala da JOA $(\alpha=0,001)$, portanto neste grupo, a cirurgia não produz os resultados esperados, segundo este estudo. A análise das médias não demonstra que estes evoluem com piora progressiva, significativa estatisticamente, no período pós operatório, apesar dos escores negativos, uma vês que estes não são menores que z crítico para piora $-3,29$ e $\alpha=0,001$, porém isso não deve ser interpretado como um sinal que houve estabilização no quadro.

\section{Dor cervical (Tabelas 13, 14, 15 e 16))}

A dor cervical ocorre quando alterações degenerativas estimulam nociceptores intrínsecos da coluna cervical. É bilateral e pode ser acompanhada de dor referida ou radicular em um dos membros superiores ou de dor interescapular. A dor cervical é um sintoma presente em $62,5 \%$ dos casos analisados no estudo (Tabela 13).

Tabela 13. Presença de dor cervical e evolução operatória.

\begin{tabular}{c|c|c|c|c}
\hline & $\mathbf{f}$ & JOA Pré & JOA Pós & JOA Tardio \\
\hline $\operatorname{Sim}$ & 125 & 9,6 & 12,63 & 12,64 \\
\hline Não & 75 & 9,66 & 12,49 & 12,24 \\
\hline
\end{tabular}

$\mathrm{X}-\mathrm{X}^{1}=3 / \mathrm{X}-\mathrm{X}^{2}=3,01$

$X-X^{1}=2,86 / X-X^{2}=2,61$

$3 / 2,48 / 11,18=3 / 0,22=13,63 / / / / 3,01 / 2,48 / 11,18=3,01 / 0,22=13,68$ $2,86 / 2,48 / 8,66=2,86 / 0,286=10 / / / / 2,61 / 2,48 / 8,66=2,61 / 0,286=9,12$ nível de significância $\alpha=0,001$ e z crítico 3,29 $/ \delta=2,48 / X=9,63$.
Tabela 14. Idade e período de evolução pré-operatório

\begin{tabular}{c|c|c|c}
\hline & $\mathbf{f}$ & Idade & Períod Evoluç Pré-0p. \\
\hline Sim & 125 & 50,97 & 18,9 \\
\hline Não & 75 & 54,81 & 18,10 \\
\hline Intervalo de confiança \\
\hline Sim & $52,41 \pm 3,59(48,82-56)$ e $(14,13-23,07)$ \\
\hline Não & $52,41 \pm 4,65(47,76-57,05)$ e $(12,83-24,37)$ \\
\hline
\end{tabular}

A presença, ou não, de dor cervical pré-operatória não se correlaciona com o prognóstico de melhora neurológica.

Instabilidade radiográfica (Tabelas 15 e 16)

Tabela 15. Instabilidade e evolução operatória.

\begin{tabular}{c|c|c|c|c}
\hline & $\mathbf{f}$ & JOA Pré & JOA Pós & JOA Tardio \\
\hline $\operatorname{Sim}$ & 20 & 8,65 & 12,35 & 12,5 \\
\hline Não & 180 & 9,73 & 12,60 & 12,49 \\
\hline
\end{tabular}

$X-X^{1}=2,72 / X-X^{2}=2,87$

$X-X^{1}=2,97 / X-X^{2}=2,86$

$2,72 / 2,48 / 4,47=2,72 / 0,55=4,94 / / / / 2,87 / 2,48 / 4,47=2,87 / 0,55=5,21$

$2,97 / 2,48 / 13,41=2,97 / 0,184=16,14 / / / / 2,86 / 2,48 / 13,41=2,86 / 0,184=15,54$ nível de significância $\alpha=0,001$ e z crítico 3,29 $/ \delta=2,48 / X=9,63$

Tabela 16. Idade e evolução pré-operatória.

\begin{tabular}{c|c|c|c}
\hline & $\mathbf{f}$ & Idade & Períod. Evolução Pre-0p. \\
\hline $\operatorname{Sim}$ & 20 & 52,2 & 19,35 \\
\hline Não & 180 & 52,43 & 18,52 \\
\hline
\end{tabular}

Intervalo de confiança

\begin{tabular}{l|l}
\hline Sim & $52,41 \pm 9(43,41-61,41)$ e $18,6 \pm 11,18(7,42-29,78)$ \\
\hline Não & $52,41 \pm 3(49,41-55,41)$ e $18,6 \pm 3,73(14,87-22,33)$ \\
\hline
\end{tabular}

A observação, ou não, de instabilidade radiológica pré-operatória não se correlaciona com a predileção de melhora neurológica, ou não, pós-cirúrgica.

\section{Inversão da lordose (Tabelas 17 e 18)}

Tabela 17. Inversão da lordose e evolução operatória.

\begin{tabular}{c|c|c|c|c}
\hline & $\mathbf{F}$ & JOA Pré & JOA Pós & JOA Tardio \\
\hline $\operatorname{Sim}$ & 43 & 8,93 & 11,34 & 11,53 \\
\hline Não & 157 & 9,82 & 12,91 & 12,75 \\
\hline
\end{tabular}

$X-X^{1}=1,71 / X-X^{2}=1,9$

$X-X^{1}=3,28 / X-X^{2}=3,12$

$1,71 / 2,48 / 6,5=1,71 / 0,38=4,5 / / / / 1,9 / 2,48 /=1,9 / 0,38=5$

$3,28 / 2,48 / 12,52=2,86 / 0,2=14,3 / / / / 3,12 / 2,48 / 12,52=3,12 / 0,2=15,6$

nível de significância $\alpha=0,001$ e z crítico 3,29 $/ \delta=2,48 / X=9,63$

Tabela 18. Idade e período de evolução pré-operatória.

\begin{tabular}{c|c|c|c}
\hline & $\mathbf{f}$ & Idade & Período Evoluç. Pré-0p. \\
\hline $\operatorname{Sim}$ & 43 & 54,83 & 21,41 \\
\hline Não & 157 & 51,75 & 17,83 \\
\hline
\end{tabular}

Intervalo de confiança

\begin{tabular}{c|l}
\hline Sim & $52,41 \pm 6,19(46,22-58,6)$ e $18,6 \pm 7,69(10,91-26,29)$ \\
\hline Não & $52,41 \pm 3,21(49,2-55,62)$ e $18,6 \pm 4(14,6-22,6)$ \\
\hline
\end{tabular}


A ocorrência de alterações da curvatura cervical ao Rx, com inversãoda lordose fisiológica, pré-cirúrgico não se correlaciona com o prognóstico de melhora neurológica.

\section{Compressão em múltiplos níveis na RNM pré-operatória (Tabelas} 19 e 20)

Tabela 19. Compressão em múltiplos níveis na RNM e evolução operatória.

\begin{tabular}{c|c|c|c|c}
\hline & $\mathbf{f}$ & JOA Pré & JOA Pós & JOA Tardio \\
\hline Sim & 104 & 9,59 & 11,83 & 11,89 \\
\hline Não & 96 & 9,66 & 13,38 & 13,14 \\
\hline
\end{tabular}

$X-X=2,2 / 2,26$

$\mathrm{X}-\mathrm{X}=3,75 / 3,51$

$2,2 / 2,48 / 10,19=2,2 / 0,24=9,16 / / / / 2,26 / 2,48 / 10,19=2,26 / 0,24=9,41$

$3,75 / 2,48 / 9,79=3,75 / 0,25=15 / / / / 3,51 / 2,48 / 9,79=3,51 / 0,25=14,04$

nível de significância $\alpha=0,001$ e z crítico 3,29 $/ \delta=2,48 / X=9,63$.

Tabela 20. Idade e evolução pré-operatória.

\begin{tabular}{c|c|c|c}
\hline & $\mathbf{f}$ & Idade & Períod. Evolução Pre-0p. \\
\hline $\operatorname{Sim}$ & 104 & 56,29 & 21,54 \\
\hline Não & 96 & 48,20 & 15,41 \\
\hline
\end{tabular}

Intervalo de confiança

\begin{tabular}{l|l}
\hline Sim & $(48,47-56,35)$ e $(13,7-23,50)$ \\
\hline Não & $(48,31-56,51)$ e $(13,5-23,7)$ \\
\hline
\end{tabular}

No caso da variável compressão em múltiplos níveis não há diferenças significativas nos resultados obtidos segundo a pontuação na escala da JOA com $\alpha=0,001$, entre os períodos pré-operatório e pós-operatório precoce e tardio, porém observa-se melhora muito superior na pontuação no grupo onde há compressões pontuais. Neste caso, a média de idade é abaixo do limite inferior do intervalo de confiança, fator prognóstico positivo.

Nível primário da compressão (Tabela 21)

Tabela 21. Nível primário da compressão e evolução pós-operatória.

\begin{tabular}{c|c|c|c|c}
\hline & $\mathbf{f}$ & JOA Pré & JOA Pós & JOA Tardio \\
\hline C2 - C3 & 1 & 13 & 15 & 15 \\
\hline C3 - C4 & 24 & 10,12 & 12,20 & 12,58 \\
\hline C4 - C5 & 30 & 9,31 & 12,34 & 12,55 \\
\hline C5-C6 & 30 & 8,81 & 12,92 & 11,86 \\
\hline C6-C7 & 10 & 9,4 & 12,7 & 12,9 \\
\hline C7 - T1 & 1 & 10 & 11 & 11 \\
\hline Múltiplos & 104 & 9,59 & 11,83 & 11,89 \\
\hline
\end{tabular}

$X-X^{1}=5,37 / X-X^{2}=5,37$

$X-X^{1}=2,57 / X-X^{2}=2,95$

$X-X^{1}=2,71 / X-X^{2}=2,92$

$X-X^{1}=3,29 / X-X^{2}=2,23$

$\mathrm{X}-\mathrm{X}^{1}=3,07 / \mathrm{X}-\mathrm{X}^{2}=3,27$

$X-X^{1}=1,37 / X-X^{2}=1,37$

$X-X^{1}=2,2 / X-X^{2}=2,26$

$5,37 / 2,48 / 1=5,37 / 2,48=2,16 / / / / 5,37 / 2,48 / 1=5,37 / 2,48=2,16$ $2,57 / 2,48 / 4,89=2,57 / 0,507=5,06 / / / / 2,95 / 2,48 / 4,89=2,95 / 0,507=5,81$ $2,71 / 2,48 / 6,16=2,71 / 0,402=6,77 / / / / 2,92 / 2,48 / 6,16=2,92 / 0,402=7,3$ $3,29 / 2,48 / 6,16=3,29 / 0,402=8,22 / / / / 2,23 / 2,48 / 6,16=2,23 / 0,402=5,57$ $3,07 / 2,48 / 3,16=3,07 / 0,784=3,91 / / / / 3,27 / 2,48 / 3,16=3,27 / 0,784=4,17$ $1,37 / 2,48 / 1=1,37 / 2,48=0,55 / / / / 1,37 / 2,48 / 1=1,37 / 2,48=0,55$

$2,2 / 2,48 / 10,19=2,2 / 0,24=9,16 / / / / 2,26 / 2,48 / 10,19=2,26 / 0,24=9,41$

nível de significância $\alpha=0,001$ e z crítico 3,29 $/ \delta=2,48 / X=9,63$.
Não se observa correlação entre o nível, ou seja, a altura da compressão e o resultado final.

Presença de hipersinal em T1/T2 na RNM pré-operatória (Tabela 22)

Tabela 22. Hipersinal na RNM pré-operatória e evolução operatória.

\begin{tabular}{c|c|c|c|c}
\hline & $\mathbf{f}$ & JOA Pré & JOA Pós & JOA Tardio \\
\hline $\operatorname{Sim}$ & 150 & 9,21 & 12,04 & 11,84 \\
\hline Não & 50 & 10,88 & 14,18 & 14,44 \\
\hline
\end{tabular}

$X-X^{1}=2,41 / X-X^{2}=2,21$

$\mathrm{X}-\mathrm{X}^{1}=4,55 / \mathrm{X}-\mathrm{X}^{2}=4,81$

$2,41 / 2,48 / 12,24=2,41 / 0,2=12,05 / / / / 2,21 / 2,48 / 12,24=2,21 / 0,2=11,05$ $4,55 / 2,48 / 7,07=4,55 / 0,35=13 / / / / 4,81 / 2,48 / 7,07=4,81 / 0,35=13,74$ nível de significância $\alpha=0,001$ e z crítico 3,29 $/ \delta=2,48 / X=9,63$.

Nos grupos com e sem hipersinal na RNM houve melhora estatística significativa para $\alpha=0,001$, porém no grupo sem hipersinal o desempenho de melhora da pontuação foi bem mais significativo (Tabela 23).

Tabela 23. Idade e evolução pré-operatória.

\begin{tabular}{c|c|c|c}
\hline & $\mathbf{f}$ & Idade & Períod. Evolução Pre-Op. \\
\hline $\operatorname{Sim}$ & 150 & 51,38 & 18,48 \\
\hline Não & 50 & 55,52 & 19 \\
\hline
\end{tabular}

Intervalo de confiança

\begin{tabular}{c|c}
\hline $\operatorname{Sim}$ & $(49,13-55,69)$ e $(14,52-22,68)$ \\
\hline Não & $(46,72-58,1)$ e $(11,53-25,67)$ \\
\hline
\end{tabular}

Não foi observada diferença induzida pela idade média do grupo ou pelo período de evolução da doença, que foi semelhante nos dois grupos.

\section{Síndrome Clínica de apresentação no momento do diagnóstico (Tabela 24)}

Tabela 24. Síndrome clínica e evolução operatória.

\begin{tabular}{c|c|c|c|c}
\hline & $\mathbf{f}$ & JOA Pré & JOA Pós & JOA Tardio \\
\hline Mielopatia & 133 & 9,51 & 12,44 & 12,31 \\
\hline Mielorradicul & 67 & 9,85 & 12,85 & 12,85 \\
\hline Geral & 200 & 9,63 & 12,58 & 12,49 \\
\hline
\end{tabular}

$X-X^{1}=2,81 / X-X^{2}=2,68$

$X-X^{1}=3,22 / X-X^{2}=3,22$

$\mathrm{X}-\mathrm{X}^{1}=2,95 / \mathrm{X}-\mathrm{X}^{2}=2,86$

$2,81 / 2,48 / 11,53=2,81 / 0,215=13,06 / / / / 2,68 / 2,48 / 11,53=2,68 / 0,215=12,46$ $3,22 / 2,48 / 8,18=3,22 / 0,303=10,62 / / / / 3,22 / 2,48 / 8,18=3,22 / 0,303=10,62$ $2,95 / 2,48 / 14,14=2,95 / 0,175=16,85 / / / / 2,86 / 2,48 / 14,14=2,86 / 0,175=16,34$ nível de significância $\alpha=0,001$ e z crítico 3,29 $/ \delta=2,48 / X=9,63$.

A síndrome de apresentação clínica não interfere no prognóstico de melhora neurológica.

\section{Período de evolução pré-operatório}

O período de evolução pré-operatório, compreendido entre o início dos sintomas e a cirurgia primária, teve máximo de 90 meses e mínimo de 01 mês, com média aritmética de 18,6 meses, mediana e modo de 12 meses, desvio padrão de 15,2 meses e distorção de 1,63 meses (Tabelas 25 e 26). 
Tabela 25. Período de evolução pré-operatório e evolução operatória.

\begin{tabular}{c|c|c|c|c}
\hline & $\mathbf{f}$ & JOA Pré & JOA Pós & JOA Tardio \\
\hline $1+6$ & 17 & 7,88 & 13,64 & 13,76 \\
\hline $6+12$ & 56 & 10 & 13,83 & 13,48 \\
\hline $12+18$ & 44 & 9,86 & 12,61 & 12,59 \\
\hline $18+24$ & 22 & 9,63 & 12,63 & 12,63 \\
\hline $24+30$ & 23 & 9,6 & 11,21 & 10,91 \\
\hline $30+36$ & 9 & 8,22 & 9,44 & 9,66 \\
\hline $36+42$ & 10 & 10,4 & 12,2 & 12 \\
\hline $42+$ & 19 & 9,84 & 11,10 & 11,57 \\
\hline
\end{tabular}

$X-X^{1}=4,01 / X-X^{2}=4,13$

$X-X^{1}=4,2 / X-X^{2}=3,85$

$X-X^{1}=2,98 / X-X^{2}=2,96$

$X-X^{1}=3 / X-X^{2}=3$

$X-X^{1}=1,58 / X-X^{2}=1,28$

$X-X^{1}=-0,19 / X-X^{2}=0,03$

$X-X^{1}=2,57 / X-X^{2}=2,37$

$X-X^{1}=1,47 / X-X^{2}=1,94$

$\mathrm{n}=174,01 / 2,48 / 4,12=4,01 / 0,6=6,68 / / / / 4,13 / 2,48 / 4,12=4,13 / 0,6=6,88$

$\mathrm{n}=564,2 / 2,48 / 7,48=4,2 / 0,33=12,72 / / / / 4,2 / 2,48 / 7,48=4,2 / 0,33=12,18$

$\mathrm{n}=442,98 / 2,48 / 6,63=2,98 / 0,37=8,05 / / / / 2,96 / 2,48 / 6,63=2,96 / 0,37=8$

$\mathrm{n}=22$ 3/2,48/4,69=3/0,528 = 5,68 ////3/2,48/4,69=3/0,528 = 5,68

$n=231,58 / 2,48 / 4,79=1,58 / 0,517=3,05 / / / / 1,28 / 2,48 / 4,79=1,28 / 0,517=2,47$

$\mathrm{n}=9-0,19 / 2,48 / 3=-0,19 / 0,82=-0,23 / / / / 0,03 / 2,48 / 3=0,03 / 0,82=0,036$

$\mathrm{n}=102,57 / 2,48 / 3,16=2,57 / 0,78=3,28 / / / / 2,37 / 2,48 / 3,16=2,37 / 0,78=3,03$

$\mathrm{n}=191,47 / 2,48 / 4,35=1,47 / 0,57=2,57 / / / / 1,94 / 2,48 / 4,35=1,94 / 0,57=3,4$ nível de significância $\alpha=0,001$ e z crítico 3,29 / $\delta=2,48 / X=9,63$.

Tabela 26. Período de evolução pré-operatório e evolução operatória segundo a Taxa de recuperação da JOA.

\begin{tabular}{c|c|c|c}
\hline & $\mathbf{f}$ & TX Rec Pré & TX Rec Pós \\
\hline $1+6$ & 17 & 64,54 & 65,45 \\
\hline $6+12$ & 56 & 54,10 & 48,91 \\
\hline $12+18$ & 44 & 37,54 & 38,61 \\
\hline $18+24$ & 22 & 39,42 & 38,73 \\
\hline $24+30$ & 23 & 22,09 & 16,61 \\
\hline $30+36$ & 9 & 17,42 & 20,60 \\
\hline $36+42$ & 10 & 25,69 & 26,8 \\
\hline $42+$ & 19 & 20,5 & 25,88 \\
\hline
\end{tabular}

O período de tempo, aferido emmeses, decorrido entre o início dos sintomas e a cirurgia interfere de uma maneira direta e muito significativa no resultado final de melhora neurológica. Observa-se correlação inversa entre aumento do período e melhora napontuação. Após transcorridos 24 meses de início dos sintomas não se observa melhora estatisticamente significativa com fixação $\alpha=0,001$.

Em relação à variável período de evolução pré-operatório observamos melhora estatisticamente significativa, $\operatorname{com} \alpha=0,001$, para períodos inferiores a 24 meses, porém com $\alpha=0,01$ a melhora é estatisticamente significativa para todos os grupos.

Observa-se melhores escores de melhora para os grupos com menor tempo de evolução (Figura 2).

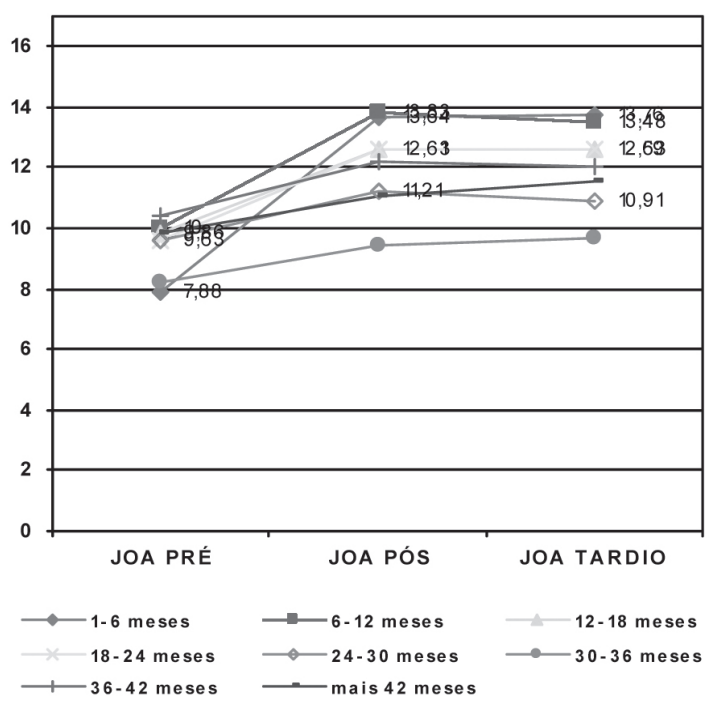

Figura 2. Período de evolução pré-operatório e evolução cirúrgica.

Status neurológico pré-operatório, segundo a escala da JOA (Figuras 3 e 4, Tabelas 27 e 28)

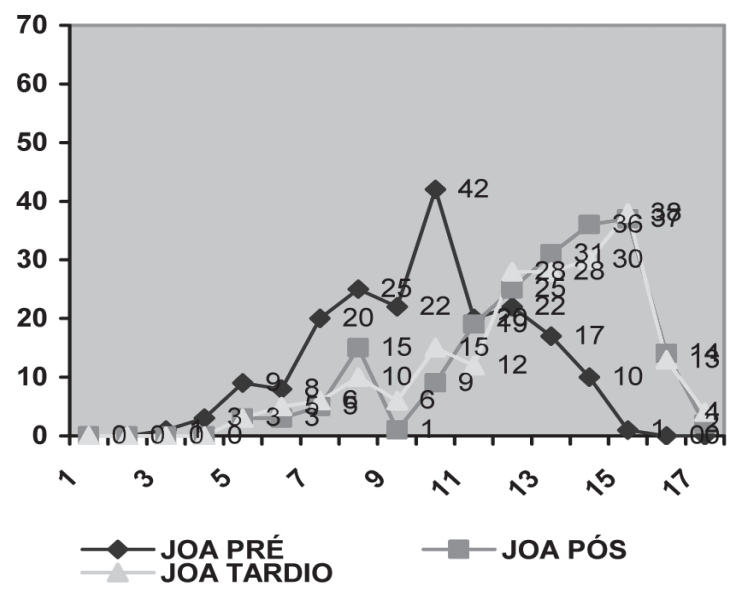

Figura 3. Distribuição da frequência percentual da pontuação segundo a escala da JOA.

Tabela 27. Pontuação pré-operatória na escala da JOA e evolução final.

\begin{tabular}{c|c|c|c|c}
\hline & $\mathbf{f}$ & JOA Pré & JOA Pós & JOA Tardio \\
\hline $3+5$ & 13 & 4,61 & 10,46 & 10,07 \\
\hline $5+7$ & 28 & 6,71 & 10,82 & 10,64 \\
\hline $7+9$ & 47 & 8,46 & 11,65 & 11,63 \\
\hline $9+11$ & 62 & 10,32 & 12,95 & 12,98 \\
\hline $11+13$ & 39 & 12,43 & 14,38 & 14,12 \\
\hline $13+15$ & 11 & 14,09 & 15 & 15,18 \\
\hline
\end{tabular}

$X-X^{1}=0,83 / X-X^{2}=0,44$

$X-X^{1}=1,19 / X-X^{2}=1,01$

$X-X^{1}=2,02 / X-X^{2}=2$

$X-X^{1}=3,32 / X-X^{2}=3,35$

$X-X^{1}=4,75 / X-X^{2}=4,49$

$X-X^{1}=5,37 / X-X^{2}=5,55$

$\mathrm{n}=13 \quad 0,83 / 2,48 / 3,6=0,83 / 0,68=1,22 / / / / / 0,44 / 2,48 / 3,6=0,44 / 0,68=0,64$

$n=28 \quad 1,19 / 2,48 / 5,29=1,19 / 0,46=2,58 / / / / 1,01 / 2,48 / 5,29=1,01 / 0,46=2,19$

$\mathrm{n}=472,02 / 2,48 / 6,85=2,02 / 0,36=5,61 / / / / 2 / 2,48 / 6,85=2 / 0,36=5,5$

$\mathrm{n}=623,32 / 2,48 / 7,87=3,32 / 0,315=10,53 / / / / 3,35 / 2,48 / 7,87=3,35 / 0,315=10,63$

$n=394,75 / 2,48 / 6,24=4,75 / 0,397=11,96 / / / / 4,49 / 2,48 / 6,24=4,49 / 0,397=11,3$

$n=11 \quad 5,37 / 2,48 / 3,31=5,37 / 0,75=7,16 / / / / 5,37 / 2,48 / 3,31=5,37 / 0,75=7,16$

nível de significância $\alpha=0,001$ e z crítico 3,29 $/ \delta=2,48 / X=9,63$. 
Tabela 28. Pontuação pré-operatória na escala da JOA e evolução final segundo à Taxa de recuperação da JOA.

\begin{tabular}{c|c|c|c}
\hline & $\mathbf{f}$ & TX Rec Pré & TX Rec Pós \\
\hline $3+5$ & 13 & 52,86 & 58,63 \\
\hline $5+7$ & 28 & 37,94 & 35,08 \\
\hline $7+9$ & 47 & 40,30 & 36,28 \\
\hline $9+11$ & 62 & 37,90 & 42,13 \\
\hline $11+13$ & 39 & 41,15 & 32,31 \\
\hline $13+15$ & 11 & 40,46 & 43,32 \\
\hline
\end{tabular}

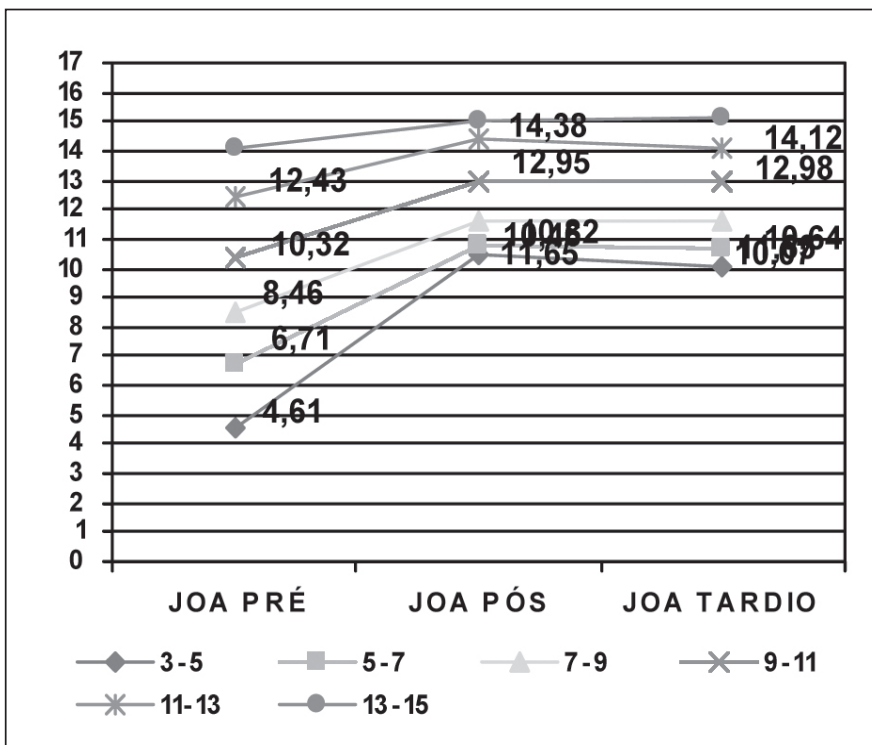

Figura 4. Evolução da pontuação na escala da JOA no período pré e pós-cirúrgico.

Em relação a variável pontuação pré-operatória segundo a escala da JOA observamos que nos grupos com pontuação entre 3 e 7 não há melhora estatisticamente significativa entre os períodos pré e pós operatório para $\alpha=0,001$, sendo que para o grupo entre 5 e 7 há melhora com $\alpha=0,05$, indicando que o status neurológico pré-operatório é fator preditivo de melhora pós operatória, com associação direta entre a pontuação na escala da JOA, sendo que quanto menor esta, menor a melhora (Tabela 29).

Tabela 29. Idade e período de evolução pré-operatório.

\begin{tabular}{c|c|c|c}
\hline & f & Idade & Período de Evoluç. Pré-Op. \\
\hline & & & 12,61 \\
\hline $3+5$ & 13 & 45,76 & 17,35 \\
\hline $5+7$ & 28 & 52,92 & 22,27 \\
\hline $7+9$ & 47 & 54,23 & 16,35 \\
\hline $9+11$ & 62 & 53,04 & 19,28 \\
\hline $11+13$ & 39 & 50,76 & 23,45 \\
\hline $13+15$ & 11 & 53,45 & \\
\hline Intervalo de confiança \\
\hline $3+5$ & $52,41 \pm 11,17(41,24-63,7)$ e $18,6 \pm 13,9(4,7-32,5)$ \\
\hline $5+7$ & $52,41 \pm 7,6(44,81-60,01)$ e $18,6 \pm 9,45(9,15-28,05)$ \\
\hline $7+9$ & $52,41 \pm 5,87(46,54-58,28)$ e $18,6 \pm 7,3(11,3-25,9)$ \\
\hline $9+11$ & $52,41 \pm 5,11(47,3-57,52)$ e $18,6 \pm 6,35(12,25-24,95)$ \\
\hline $11+13$ & $52,41 \pm 6,44(45,97-58,85)$ e $18,6 \pm 8,01(10,59-26,61)$ \\
\hline
\end{tabular}

Não observamos interferência de discrepâncias entre período de evolução e média de idade dos grupos.

\section{Segmento pós-operatório (Tabela 30, Figura 5)}

Tabela 30. Período de seguimento pós-operatório.

\begin{tabular}{c|c|c|c|c}
\hline & $\mathbf{f}$ & $\mathbf{f r}$ & $\mathrm{Fr}$ & $\%$ \\
\hline+24 & 29 & 0,145 & 0,145 & 14,5 \\
\hline $24+48$ & 79 & 0,395 & 0,54 & 39,5 \\
\hline $48+$ & 92 & 0,46 & 1,0 & 46 \\
\hline$\Sigma$ & 200 & 1,00 & 1,00 & 100 \\
\hline
\end{tabular}

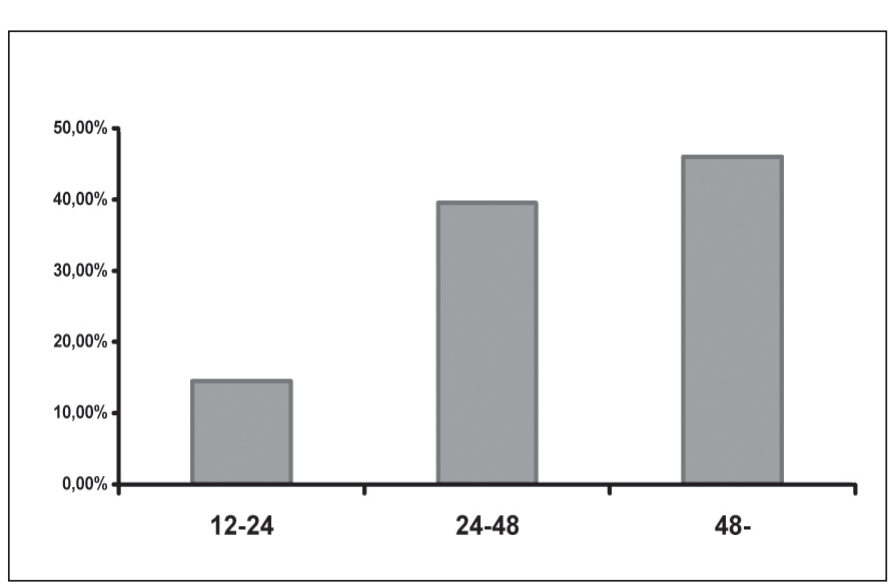

Figura 5. Distribuição percentual da média de seguimento pós-operatório.

O segmento mínimo para inclusão no trabalho foi de 24 meses. No total $46 \%$ dos casos tiveram segmento superior a 48 meses.

Resultado final á longo prazo (Tabela 31)

Tabela 31. Resultado final á longo prazo.

\begin{tabular}{c|c|c|c}
\hline & $\mathbf{f}$ & $\mathbf{f r}$ & $\%$ \\
\hline Melhora & 160 & 0,8 & 80 \\
\hline Inalterado & 28 & 0,14 & 14 \\
\hline Piora Tardia & 12 & 0,06 & 6 \\
\hline$\Sigma$ & 200 & 1,00 & 100 \\
\hline
\end{tabular}

No total $80 \%$ dos casos apresentaram melhora estatisticamente significativa (Figura 6).

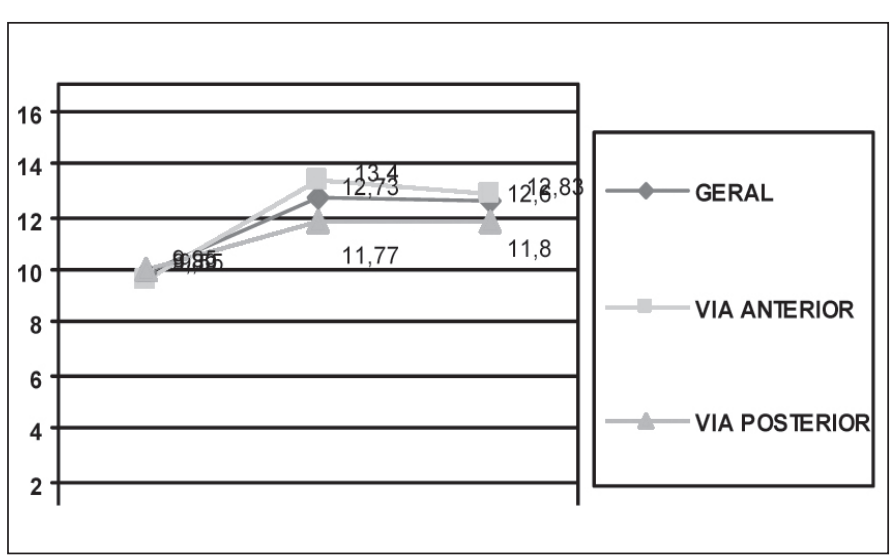

Figura 6. Evolução final em pontos de acordo com a escala da JOA. 
Menor espessura ântero-posterior do canal vertebral (Tabela 32)

Tabela 32. Espessura do canal vertebral antes a após a cirurgia.

\begin{tabular}{c|c|c}
\hline Espessura do canal & Pré op & Pós op \\
\hline Geral & 7,64 & 10,93 \\
\hline Melhora & 7,59 & 11,02 \\
\hline Inalterado & 7,16 & 10,66 \\
\hline Piora & 9,33 & 9,8 \\
\hline Via anterior & 7,62 & 10,93 \\
\hline Via posterior & 7,76 & 10,93
\end{tabular}

Tanto a via anterior como a posterior foram eficazez em produzir aumento do diâmetro AP do canal, e de sua área. (Tabela 33).

Tabela 33. Espessura do canal vertebral e evolução final segundo a escala da JOA.

\begin{tabular}{c|c|c|c|c}
\hline Espessura do canal $(\mathbf{m m})$ & $\mathbf{n}$ & JOA Pré & JOA Pós & JOA Tardio \\
\hline $4+6$ & 10 & 6,8 & 10,1 & 10,6 \\
\hline $6+8$ & 48 & 9,5 & 12,83 & 13,02 \\
\hline $8+10$ & 45 & 9,93 & 13,6 & 13,68 \\
\hline $10+12$ & 17 & 11,76 & 14,23 & 14,05 \\
\hline Geral & 120 & 9,75 & 13,09 & 13,21 \\
\hline
\end{tabular}

$X-X^{1}=0,35 / X-X^{2}=0,85$

$X-X^{1}=2,98 / X-X^{2}=3,27$

$X-X^{1}=3,85 / X-X^{2}=3,93$

$X-X^{1}=4,48 / X-X^{2}=4,3$

$\mathrm{n}=100,35 / 2,48 / 3,16=0,35 / 0,78=0,44 / / / / 0,85 / 2,48 / 3,16=0,85 / 0,78=1,08$

$\mathrm{n}=482,98 / 2,48 / 6,92=2,98 / 0,36=8,27 / / / / 3,27 / 2,48 / 6,92=3,27 / 0,36=9,08$

$\mathrm{n}=453,85 / 2,48 / 6,7=3,85 / 0,37=10,4 / / / / 3,93 / 2,48 / 6,7=3,93 / 0,37=10,62$

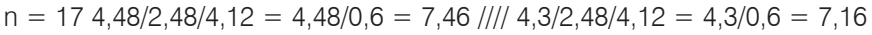

nível de significância $\alpha=0,001$ e z crítico 3,29 / $\delta=2,48 / X=9,63$.

A análise da variável espessura do canal evidencia que pacientes com canais com espessura de 06 mm de diâmetro, ântero-posterior, ou menos, não apresentam melhora estatisticamente significativa para $\alpha=0,001$ entre os períodos pré e pós operatórios.

Ainda podemos associar a espessura do canal ao status neurológico pré-operatório, sendo que quanto menor a área do canal, pior o status neurológico (Figura 7).

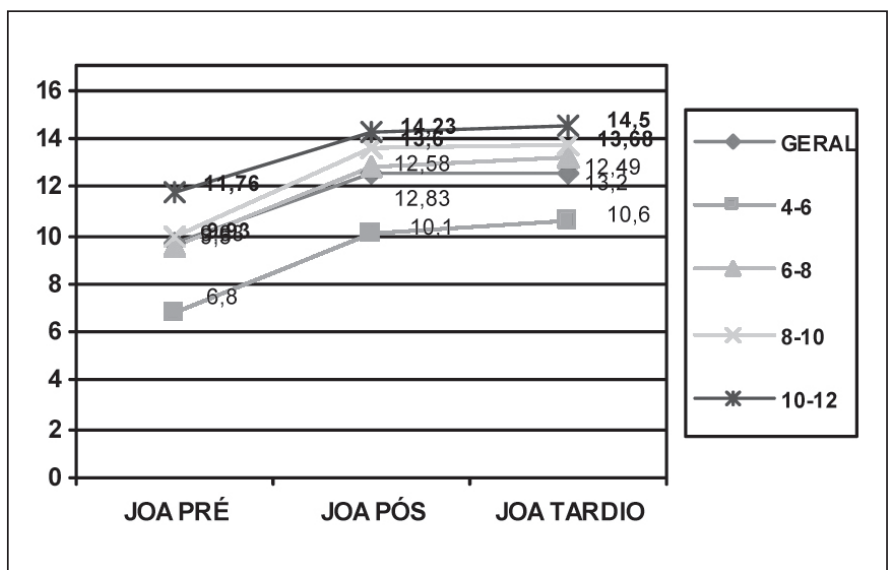

Figura 7. Espessura do canal nos períodos pré-operatório e evolução segundo pontuação na escala da JOA.

\section{DISCUSSÃO}

No estudo foi observado evidente predomínio de acometimento dos pacientes do sexo masculino, enquanto a literatura mostra evidente predomínio do feminino, o que pode ser relacionados a fatores peculiares da população atendida no HCFM-USP e a fatores ocupacionais em nosso meio, como os trabalhos braçais no campo e na construção civil, sem qualquer emprego de fatores ergonômicos de proteção. Há evidente predomínio da doença em grupos de trabalhadores braçais em serviços gerais e de ofício, sem especialização, com alta demanda de atividade física e exposição do aparelho ósseo, articular e muscular a cargas axiais e exigindo repetição de movimentos com lesão continuada destes elementos.

Idade: Em nosso estudo a idade média de incidência da doença foi de 52,41 anos, com maior incidência entre a quinta e sexta décadas de vida. A literatura traz relatos de maior incidência na quinta e sexta décadas de vida, produzidas na maioria em países da Europa, América do Norte e Japão. A análise da variável idade evidencia incremento estatisticamente significativo $(\alpha=0,001)$ na pontuação da escala da JOA, após a cirurgia, tanto precoce como tardiamente, para os indivíduos com idade inferior a 70 anos. No grupo com idade igual ou superior a 70 anos não se observou melhora estatística após a cirurgia $(\alpha=0,05)$, observando-se ainda piora tardia. Quando a taxa de recuperação foi o fator de mensuração, observamos os piores resultados no grupo com idade maior ou igual a 70 anos, e o melhor desempenho no grupo da quarta década de vida. Observamos evidente e forte associação entre idade cronológica de vida e desempenho operatório de melhora neurológica (JOA), sendo que para cada década de vida acrescida, após os 40 anos, estima-se que pode haver um decréscimo na taxa de recuperação de 6,1\%, ou seja, de 0,6 ao ano, após os 30 anos. Para os indivíduos com mais de 70 anos, nosso estudo não mostrou produção do resultado esperado, portanto não houve melhora significativa segundo métodos estatísticos de análise de populações. Os melhores resultados são observados nos grupos operados mais jovens, com quedas no rendimento diretamente relacionadas ao aumento na idade, mesmo que diminuído a sensibilidade dos testes. Não observamos maior incidência de complicações cirúrgicas em nenhum dos grupos.

Tabagismo: No estudo identificamos forte associação entre mielopatia cervical e tabagismo, presente em 46,5\% dos casos estudados, proporção bem superior da estimada por estudos epidemiológicos para populações brasileiras em inquéritos epidemiológicos realizados em capitais brasileiras em 2009 pelo Ministério da Saúde (Estimativas sobre frequência e distribuição sócio-demográfica de fatores de risco e proteção para doenças crônicas nas capitais dos 26 estados brasileiros e no Distrito Federal em 2009 - Secretaria em Vigilância em Saúde) VIGITEL 2009, que revelou uma frequência geral na população de fumantes de 15,5\%, sendo maior no sexo masculino (19\%) e menor no sexo feminino (12,5\%), para indivíduos acima de 18 anos de idade.

Co-morbidades: A análise das médias de pontuação obtidas segundo a escala da JOA, evidencia incremento estatisticamente significativo $(\alpha=0,001)$, entre os períodos pré-pós operatórios, no grupo com co-morbidades, rejeitando-se a hipótese de que os pacientes com co-morbidades não melhoram. Adendo para o fato que pacientes com algumas co-morbidades foram excluídos, conforme citado nos métodos.

Há, entretanto evidente melhor desempenho do grupo sem co-morbidades, evidenciado por melhores escores pós-operatórios, segundo a escala da JOA e taxa de recuperação.

Há ainda notória diferença, com nível de significância estatístico ( $\alpha=0,001)$, entre as médias de idade dos grupos, sendo que no grupo das co-morbidades, está acima do limite superior do intervalo de confiança, contribuindo para o pior desempenho.

Não houve maior incidência de complicações cirúrgicas no grupo com co-morbidades.

Sinal de Hoffman: A análise estatística do grupo onde a presença do sinal de Hoffman no exame clínico pré-operatório foi observado, evidenciou incremento na pontuação pós-operatória, precoce e tardiamente, segundo a escala da JOA, com significância estatística $(\alpha=0,001)$, notadamente com melhor desempenho do grupo onde o sinal não está presente.

Atrofia muscular: A análise estatística das médias de pontuação obtidas pela escala da JOA evidencia incremento na pontuação pós-operatória, precoce e tardia, com significância estatística 
( $\alpha=0,001)$, somente no grupo sem atrofia muscular. No grupo com atrofia muscular não há evidência estatística de incremento na pontuação pós-operatória (JOA) mesmo diminuindo a sensibilidade dos testes $(\alpha=0,05)$. Neste grupo a cirurgia não produz os resultados esperados, segundo este estudo.

Dor cervical: A presença ou não de dor cervical não afeta a evolução da doença, segundo este estudo. Não há diferenças significativas entre os grupos com ou sem dor, sendo que há melhora estatisticamente significativa $(\alpha=0,001)$ pós-cirúrgica nas médias de pontuação na escala da JOA.

Instabilidade no Rx pré-operatória: A presença ou não de instabilidade ao Rx, estático ou dinâmico pré-operatório, não afeta a evolução da doença. Não há diferenças significativas entre os grupos com e sem instabilidade e há melhora estatisticamente significativa $(\alpha=0,001)$ pós-cirúrgica nas médias de pontuação na escala da JOA. Ressalva para o fato de que todos os pacientes com instabilidade diagnosticada foram tratados com fixação, pela via anterior.

Inversão da Lordose: A presença de inversão da lordose no RX pré-operatório, não afeta a evolução da doença, não havendo diferenças significativas nos resultados obtidos segundo a pontuação na escala da JOA ( $\alpha=0,001)$ entre os períodos pré-operatório e pós-operatório precoce e tardio, e nem diferenças em relação ao grupo sem.

Nível de compressão e compressão medular em múltiplos níveis: Há predominância acentuada da compressão medular em múltiplos níveis, seguida por compressões pontuais nos níveis C4C5 e C5-C6. A análise estatística das médias obtidas pela escala da JOA evidencia incremento na pontuação pós-operatória, com significância estatística $(\alpha=0,001)$, no grupo de compressões múltiplas e também no de compressões pontuais, observados em exames de RNM pré-operatórios. Porém há notadamente um melhor desempenho do grupo onde a compressão é pontual e não há compressão em múltiplos níveis.

Em relação ao nível da compressão, não há diferenças de desempenho na evolução final. Neste grupo, onde não há compressão medular em múltiplos níveis, a média de idade fica abaixo do limite inferior do intervalo de confiança, fator prognóstico positivo.

No grupo com compressões múltiplas houve maior incidência de complicações pós-operatórias relacionadas ao procedimento cirúrgico e uso de próteses, o que pode ainda ter influenciado o resultado final.

Hipersinal medular na RNM pré-operatória: A análise das médias de pontuação obtidas pela aplicação da escala da JOA evidencia incremento estatisticamente significativo $(\alpha=0,001)$ no período pós-operatório no grupo com hipersinal medular, porém há nítido melhor desempenho no grupo sem hipersinal. Ainda quando há diagnóstico de hipersinal medular na RNM pré-operatória há uma tendência a piora tardia.

Síndrome de apresentação: Não há diferença na evolução operatória entre os grupos com síndromes de apresentação de mielopatia e mielorradiculopatia, sendo observado em ambos melhora estatisticamente significativa e escores pós-operatórios semelhantes $(\alpha=0,001)$.

Período de evolução pré-operatório: Na maioria dos casos o período de evolução da doença que precede ao tratamento cirúrgico, esteve entre os 06 e 18 meses. A análise estatística das médias de pontos obtidas pela aplicação da escala da JOA, e comparativamente entre os períodos pré e pós-operatório, e taxa de recuperação da JOA evidenciam incremento estatisticamente significativo $(\alpha=$ 0,001 ) na pontuação pós-operatória e taxa de recuperação nos grupos operados antes de 24 meses. Há ainda forte associação entre o período de evolução pré-operatório e o do desempenho final, estimando-se que para cada mês acrescentado ao total do período, há uma piora de 1\% no desempenho final segundo a taxa de recuperação. Quando este período excede os 24 meses, não observamos significância estatística na melhora da pontuação pós-operatória $(\alpha=0,001)$ ou na taxa de recuperação, para todos os grupos com evolução pré-operatória superior a 24 meses.
Status neurológico pré-operatório: No estudo observamos que para os casos submetidos ao tratamento cirúrgico com pontuação pré-operatória inferior ou igual a 07 (sete) pontos, obtidos pela aplicação da escala da JOA, a análise estatística das médias evidencia que não houve incremento na pontuação pós-operatória, precoce e tardia, com significância estatística $(\alpha=0,001)$. Para os grupos com pontuação igual ou superior a 08 (oito) pontos observou-se incremento estatisticamente significativo $(\alpha=0,001)$. A análise segundo a taxa de recuperação mostra taxas de recuperação muito significativas em todos os grupos, porém muito semelhantes em todos, em torno de $40 \%$, com pequenas variações entre os subgrupos. Deve-se interpretar que para grupos com pontuação inicial inferior ou igual a sete, mesmo apresentando melhora muito expressiva segundo a taxa de recuperação, estes partiram de uma pontuação muito baixa e a melhora na pontuação pós-cirúrgica não acrescentou o suficiente para atingir médias populacionais esperadas para os grupos, permanecendo com somas inferiores ao limite inferior do intervalo de confiança.

Assim, para os grupos com pontuação igual ou inferior a sete na escala da JOA, segundo a avaliação inicial pré-operatória, a cirurgia não produz o resultado esperado de melhora neurológica, tendo a população geral de pacientes com a doença como referência.

Isto não indica que a cirurgia não deve ser praticada nestes casos, e sim motiva a realização de estudos prospectivos para a avaliação da qualidade de vida em longo prazo neste grupo de pacientes.

\section{Resultado final á longo prazo}

Segundo o estudo 80\% dos pacientes evoluíram com melhora neurológica, mantida por longo prazo, segundo a escala da JOA. Em média houve melhora de 3,22 pontos precocemente e 3,11 pontos tardiamente (JOA pré-op $=9,55 \pm 2,51$ / JOA pós-op precoce $=12,78 \pm 2,54$ / JOA pós-op tardio $=12,66 \pm 2,66$ ) e com taxa de recuperação de 42,79 \pm 28,2 e 41,4 \pm 31,36. Em 14\% dos casos houve estabilização do quadro, porém os pacientes não evoluíram com nenhum tipo de melhora neurológica, avaliada pela escala da JOA. Na análise 6\% dos casos evolui com piora neurológica e nestes não identificamos associação com qualquer fator clínico, social ou ambiental ou com qualquer achado de exame de imagem com o resultado final. Portanto a cirurgia não influencia no resultado final nestes casos, talvez já pré-determinados a piora.

\section{Menor espessura AP do canal vertebral}

No estudo observamos que para os casos submetidos ao tratamento cirúrgico com diâmetro AP do canal vertebral inferior ou igual a 06 (seis) mm, aferidos na RNM pré-operatória, a análise estatística das médias obtidas pela escala da JOA, nos períodos pré e pós-operatório, evidencia que não houve incremento na pontuação pós-operatória, precoce e tardia, com significância estatística $(\alpha=0,001)$. Nos demais grupos houve melhora neurológica.

Via de acesso cirúrgico: Entre os grupos determinados pela via de abordagem cirúrgica, anterior ou posterior, observou-se melhora estatisticamente significativa para ambos entre os períodos pré-pós operatório, seja precoce ou tardio, com $\alpha=0,001$. Porém observa-se notadamente melhor desempenho nos escores da via anterior.

Observa-se que a média de idade do grupo da via posterior está acima do limite superior do intervalo de confiança, implicando em um envolvimento de outro fator preditivo de mau prognóstico.

\section{CONCLUSÃO}

No total $80 \%$ dos casos evoluíram com melhora, $14 \%$ com estabilização e 6\% com piora.

A piora neurológica não foi associada com nenhum fator clínico, ambiental ou de imagem.

Observou-se associação entre idade no momento da cirurgia, co-morbidade, sinal de Hoffman no exame pré-operatório, atrofia muscular, hipersinal medular na RNM pré-operatória, período de evolução pré-operatório, multiplicidade de compressões medulares 
na RNM pré-operatória, status neurológico pré-operatório e diâmetro AP do canal medular com desempenho operatório de melhora neurológica, sendo que estes fatores limitam a melhora, porém não a impedem nem estão associados com piora.

A presença de dor cervical, instabilidade radiológica pré-operatória, alteração da curvatura cervical no Rx, o nível da compressão e a síndrome de apresentação clínica não influenciam no resultado final.

No estudo idade igual ou superior a 70 anos não foi associado a nenhum tipo de melhora estatística. Identificamos associação entre aumento na idade e piora na taxa de recuperação na proporção de 0,6\% para cada ano, a partir dos 30 anos.

Período de evolução pré-operatório superior a 24 meses não foi associado com melhora. Melhores resultados estão associados menores períodos de evolução, sendo que para cada mês acrescido ao total de tempo estima-se uma redução em 1\% na taxa de recuperação.

O estudo da população com atrofia muscular não evidenciou melhora neurológica.

Pontuação pré-operatória, segundo a escala da JOA, igual ou inferior a sete pontos, apesar de apresentar taxa de recuperação muito significativa, não apresenta melhora neurológica estatisticamente significativa em relação à observada para a população geral, segundo a escala da JOA.

Espessura AP do canal igual ou inferior a seis $\mathrm{mm}$ não está associado com melhora neurológica.

Identificamos forte associação entre tabagismo e mielopatia, presente em 46,5\% no estudo, taxa muito superior ao encontrado na população geral.

\section{REFERÊNCIAS}

1. Clarke E, Robinson PK. Cervical myelopathy: a complication of cervical spondylosis. Brain. 1956;79(3):483-510

2. McCormack BM, Weinstein PR. Cervical spondylosis. An update. West J Med. 1996;165(1-2):43-51.

3. Young WF. Cervical spondylotic myelopathy: a common cause of spinal cord dysfunction in older persons. Am Fam Physician. 2000;62(5):1064-70, 1073.

4. Moore AP, Blumhardt LD. A prospective survey of the causes of non-traumatic spastic paraparesis and tetraparesis in 585 patients. Spinal Cord. 1997;35(6):361-7.

5. Montgomery DM, Brower RS. Cervical spondylotic myelopathy. Clinical syndrome and natural history. Orthop Clin North Am. 1992;23(3):487-93

6. Teresi LM, Lufkin RB, Reicher MA, Moffit BJ, Vinuela FV, Wilson GM, et al. Asymptomatic degenerative disk disease and spondylosis of the cervical spine: MR imaging. Radiology. 1987;164(1):83-8.

7. Parke WW. Correlative anatomy of cervical spondylotic myelopathy. Spine. 1988:13(7):831-7.

8. Matsunaga S, Sakou T, Taketomi E, Yamaguchi M, Okano T. The natural course of myelopathy caused by ossification of the posterior longitudinal ligament in the cervical spine. Clin Orthop Relat Res. 1994:(305):168-7.

9. Bohlman HH. Cervical spondylosis and myelopathy. Instr Course Lect. 1995:44:81-97.

10. Fehlings MG, Skaf G. A review of the pathophysiology of cervical spondylotic myelopathy with insights for potential novel mechanisms drawn from traumatic spinal cord injury. Spine. 1998:23(24):2730-7.

11. Wilkinson M. The morbid anatomy of cervical spondylosis and myelopathy.Brain. 1960;83:589-617.

12. Small JM, Dillin WH, Watkins RG. Clinical syndromes in cervical myelopathy. In: Herkowitz H, Garfin SR, Balderson RA, et al, editors. The Spine. 4th ed. Philadelphia: W.B. Saunders;1999. p. 465-74

13. Penning $L$, Wilmink JT, van Woerden $H H$, Knol E. CT myelographic findings in degenerative disorders of the cervical spine: clinical significance. AJR Am J Roentgenol. 1986;146(4):793-801.

14. Boni M, Denaro V (1987). Anatomo-clinical correlations in cervical spondylosis. J. Pediat. Orthop. 7:428-35 zo. Springer. Berlin Heidelberg New York, pp. 3-20.

15. Nurick $\mathrm{S}$. The pathogenesis of the spinal cord disorder associated with cervical spondylosis. Brain. 1972;95(1):87-100

16. Alexander JT. Natural history and nonoperative management of cervical spondylosis. In: Menezes AH, Sonntag VK, eds. Principles of spinal surgery. New York: McGraw-Hill Companies, Health Professions Division; 1996. p. 547-57.

17. Matz PG. Does nonoperative management play a role in the treatment of cervical spondylotic myelopathy? Spine J. 2006:6(6 Suppl):175S-181S.

18. Baron EM, Young WF. Cervical spondylotic myelopathy: a brief review of its pathophysiology, clinical course, and diagnosis. Neurosurgery. 2007 ;60(1Supp1 1):S35-41.

19. Symon L, Lavender P. The surgical treatment of cervical spondylotic myelopathy. Neurology. 1967; 17(2):117-27

20. Jumah KB, Nyame PK. Relationship between load carrying on the head and cervical spondylosis in Ghanaians. West Afr J Med. 1994;13(3):181-2.

21. Olive PM, Whitecloud TS 3rd, Bennett JT. Lower cervical spondylosis and myelopathy in adults with Down's syndrome. Spine. 1988;13(7):781-4.

22. Yamada $Y$, Okuizumi $H$, Miyauchi A, Takagi $Y$, Ikeda K, Harada A. Association of transforming growth factor beta1 genotype with spinal osteophytosis in Japanese women. Arthritis Rheum. 2000;43(2):452-60

23. Yoo K, Origitano TC. Familial cervical spondylosis. Case report. J Neurosurg 1998:89(1):139-41.

24. Hadley MN, Reddy SV. Smoking and the human vertebral column: a review of the impact of cigarette use on vertebral bone metabolism and spinal fusion. Neurosurgery. 1997;41(1):116-24.

25. Schimandle JH, Heller JG. Nonoperative treatment of degenerative cervical disk disease. J South Orthop Assoc. 1996 Fall;:5(3):207-12.

26. Crandall PH, Batzdorf U. Cervical spondylotic myelopathy. J Neurosurg. 1966;25(1):57-66.

27. Emery SE. Cervical spondylotic myelopathy: diagnosis and treatment. J Am Acad Orthop Surg. $2001 ; 9(6): 376-88$.

28. Epstein JA, Epstein NE. The surgical management of cervical spinal stenosis, spondylosis, and myeloradiculopathy by means of the posterior approach. In: Sherk HH, Dunn EJ, Eismont FJ, et al., editors. The cervical spine, 2nd ed. Philadelphia: J.B. Lippincott; 1989. p. 625-43.

29. McCormick WE, Steinmetz MP, Benzel EC. Cervical spondylotic myelopathy: make the difficult diagnosis, then refer for surgery. Cleve Clin J Med. 2003;70(10):899-904.

30. White AP, Biswas D, Smart LR, Haims A, Grauer JN. Utility of flexion-extension radiographs in evaluating the degenerative cervical spine. Spine. 2007;32(9):975-9. 